\section{Orally delivered SiRNA}

\section{By Brian Moy, Staff Writer}

A group at the University of Massachusetts Medical School has developed an encapsulated formulation of small interfering RNA that allows for oral delivery of the therapeutics and directs the siRNAs to their target cells. ${ }^{1}$ The technology has the potential to prevent unwanted immune responses that can occur with naked siRNA and may offer a convenient advantage over the injectable delivery systems being pursued commercially.

Although the oral delivery vehicle needs to be standardized before it can be used in the clinic, the technology is already in the hands of a biotech, RXi Pharmaceuticals Corp., which is using it to develop oral RNAi-based therapies for inflammatory diseases.

To engineer the delivery vehicle, the UMass researchers first selected a suitable target cell type-macrophages-and then worked backward to arrive at the best approach that would allow for oral dosing. The group started with empty $\beta 1,3$-D-glucan shells as a way to gain access to macrophages. In macrophages of the gut-associated lymphatic tissue, the immune cells phagocytose these particles via $\beta 1,3$-D-glucan receptor-mediated pathways. ${ }^{2}$

Layers of cationic polyethylenimine were then loaded into the shells, and anionic siRNA was bound between those layers. The result was a glucan-encapsulated siRNA particle, called GeRP.

GeRPs could be taken up by cells in the intestinal wall and subsequently engulfed by macrophages in the gut-associated lymphatic tissue. Upon phagocytosis of GeRPs by macrophages, the acidic $\mathrm{pH}$ in the phagosomes promoted release of siRNA through the porous GeRP wall.

Importantly, macrophages could migrate away from the gut and infiltrate other tissues in the body $y^{3}$ in which silencing of diseasecausing genes may be required.

As proof of concept, the UMass researchers developed GeRPs against MAP4K4, an intracellular protein kinase found in macrophages that facilitates signaling of the proinflammatory cytokine tumor necrosis factor- $\alpha$ (TNF- $\alpha$ ).

Oral delivery of GeRPs containing as little as $20 \mu \mathrm{g} / \mathrm{kg}$ of Map4k4 siRNA protected mice from lipopolysaccharide (LPS)-induced death. The mice also showed lower levels of TNF- $\alpha$ in macrophages recovered from the peritoneum, spleen, liver and lung than mice that received GeRPs containing scrambled control siRNA.
"Our method represents an important advance in addressing major challenges in delivering RNAi, not only because the siRNA is encapsulated and given orally, but also because its effects are restricted to a specific target cell type," said Michael Czech, chair of the program in molecular medicine at UMass Medical School and corresponding author on the paper in Nature that described the results.

He added that the in vivo potency of $20 \mu \mathrm{g} / \mathrm{kg}$ of orally delivered siRNA was up to 250 times greater than that of systemically delivered siRNAs.

\section{Beyond mice}

"While the results described in the paper are encouraging, there is still more to do, including corroborating these data and testing in higher species," said Antonin de Fougerolles, VP of research, immunology, metabolic and viral disease at Alnylam Pharmaceuticals Inc. "We are also interested in better understanding the delivery mechanism and the safety profile" of the GeRPs.

Klaus Giese, CSO of Silence Therapeutics plc, agreed that studies in larger animals will be important to provide data on how well the GeRPs can migrate from the gut to other tissue types. "Because the gut of humans is much larger and more complex than that of a mouse, we need to see if the GeRPs perform with similar efficacy and uptake in the human gut," he said.

Alnylam's ALN-RSV01, an aerosolized naked siRNA, is in a Phase II trial to treat respiratory syncytial virus (RSV) infection.

Silence's AtuRNAi-lipoplex (AtuPLEX) technology involves chemically modified siRNA designed for increased stability, activity and avoidance of immune response. It is formulated with three lipid components to allow for cellular uptake.

The company plans to start a Phase I trial this year of its Atu027 to treat solid tumors. The compound is an siRNA lipoplex targeting protein kinase N3 (PKN3) expression.

Czech and colleagues are conducting additional studies to optimize and standardize the delivery vehicle.

Czech is a founder and member of the scientific advisory board at $\mathrm{RXi}$, which is conducting preclinical discovery research investigating the use of oral RNAi delivery to treat rheumatoid arthritis (RA), inflammatory bowel disease (IBD), psoriasis and diabetes.

According to the UMass Medical School Office of Technology Management, a patent application has been filed for technology related to glucan shells and their ability to encapsulate and deliver various materials. RXi has exclusive worldwide rights to the technology in the field of orally delivered RNAi therapeutics; the patent otherwise is available for licensing.

A separate patent application has been filed relating to targeting MAP4K4 to attenuate systemic inflammation and is exclusively licensed to RXi. 
Moy, B. SciBX 2(20); doi:10.1038/scibx.2009.817

Published online May 21, 2009

REFERENCES

1. Aouadi, M. et al. Nature; published online April 29, 2009; doi:10.1038/nature07774

Contact: Michael Czech, University of Massachusetts Medical School, Worcester, Mass.

e-mail: michael.czech@umassmed.edu
2. Herre, J. et al. Mol. Immunol. 40, 869-876 (2004)

3. Vazquez-Torres, A. et al. Nature 401, 804-808 (1999)

COMPANIES AND INSTITUTIONS MENTIONED

Alnylam Pharmaceuticals Inc. (NASDAQ:ALNY), Cambridge, Mass.

RXi Pharmaceuticals Corp. (NASDAQ:RXII), Worcester, Mass.

Silence Therapeutics plc (LSE:SLN), London, U.K.

University of Massachusetts Medical School, Worcester, Mass. 